\title{
Heating and ultraviolet light activate anti-stress gene functions in humans
}

\author{
Victor F. Semenkov ${ }^{1 *}$, Anatoli I. Michalski ${ }^{2,3}$ and Alexander M. Sapozhnikov ${ }^{4}$ \\ ${ }^{1}$ Pirogov Russian National Research Medical University, Moscow, Russia, ${ }^{2}$ V. A. Trapeznikov Institute of Control Sciences, \\ Russian Academy of Sciences, Moscow, Russia, ${ }^{3}$ Research University - Higher School of Economics, Moscow, Russia, \\ ${ }^{4}$ Shemyakin-Ovchinnikov Institute of Bioorganic Chemistry RAS, Moscow, Russia
}

\section{OPEN ACCESS}

Edited by:

Elena G. Pasyukova, Institute of Molecular Genetics of Russian Academy of Sciences,

Russia

Reviewed by:

Anatoliy I. Yashin,

Duke University, USA

Danilo Maddalo,

Memorial Sloan Kettering Cancer

Center, USA

*Correspondence:

Victor F. Semenkov,

Pirogov Russian National Research Medical University, Ostrovitianov

Street 1, Moscow 117997, Russia vfsemenkov@mail.ru

Specialty section:

This article was submitted to Genetics of Aging

a section of the journal

Frontiers in Genetics

Received: 19 January 2015 Accepted: 03 July 2015 Published: 21 July 2015

Citation:

Semenkov VF, Michalski A and Sapozhnikov AM (2015) Heating and ultraviolet light activate anti-stress gene functions in humans.

Front. Genet. 6:245.

doi: 10.3389/fgene.2015.00245
Different environmental factors (i.e., toxins, heavy metals, ultraviolet (UV) rays, and $X$-radiation) cause damage to DNA, cell membranes and other organelles and induce oxidative stress, which results in the excessive production of reactive oxygen species (ROS) by phagocytes. All types of cell stress are accompanied by the activation of anti-stress genes that can suppress ROS synthesis. We hypothesized that different environmental factors would affect organisms through the activation of anti-stress genes by autologous serum (AS) proteins, followed by the synthesis of molecules that increase cell resistance to oxidative stress. The goal of this work was to study the influence of AS on ROS production by peripheral blood neutrophils isolated from donors in different age groups. Neutrophils were isolated from 59 donors (38-94 years old). AS was heated at $100^{\circ} \mathrm{C}$ for $30 \mathrm{~s}$. or irradiated by UV light at $200-280 \mathrm{~nm}$ and $8 \mathrm{~W}$ for $10 \mathrm{~min}$. Neutrophils were exposed to heat shock at $42^{\circ} \mathrm{C}$ for $1 \mathrm{~min}$. (short-term heating stress) or $43^{\circ} \mathrm{C}$ for 10 min., followed by the determination of the chemiluminescence reaction induced by zymosan. AS can increase or decrease ROS production by neutrophils depending on the structure of the proteins in the serum; these structures can be changed by heating or UV treatment and the temperature of their interaction ( 4 or $37^{\circ} \mathrm{C}$ ). We propose that the effect of environmental factors on AS proteins can cause an adverse increase in oxidative stress levels due to the functional reduction of anti-stress genes. We found a negative correlation between the quantity of intracellular Hsp70 and levels of intracellular ROS production following 10 min of heat shock at $43^{\circ} \mathrm{C}$. Short-term heating stress (1 min) at $42^{\circ} \mathrm{C}$ was followed by a prominent reduction in ROS production. This effect may be a result of the impact of the hormone adrenaline on the functions of anti-stress genes. Indeed, the same effect was observed after treatment of the neutrophils with adrenaline at concentrations of $10^{-4}$ and $10^{-5} \mathrm{M}$. In contrast, dexamethasone from the other stress hormone group did not evoke the same effect at the same concentrations.

Keywords: reactive oxygen species, autologous proteins, ultraviolet light, heat shock proteins, phagocytosis, short-term heating stress, adrenaline, dexamethasone

\section{Introduction}

Oxidative stress refers to the production of reactive oxygen species (ROS) in excessive concentrations in humans. Cell stress, especially oxidative stress, is one of main causes of many age-related diseases, including coronary heart diseases, cardiosclerosis, arterial hypertension, Alzheimer and Parkinson diseases, and cerebrovascular atherosclerosis (Penke et al., 2011). 
Different environmental factors (i.e., toxins, heavy metals, ultraviolet (UV) rays, and X-radiation) cause damage to DNA, cell membranes and other organelles and induce oxidative stress, which results in the production of excessive concentrations of ROS by phagocytes. Phagocytes were discovered by Ilja Mechnikov and are the main cells that produce ROS in organisms. Many studies have used cell heating to produce a stress reaction and activate anti-stress genes. Indeed, all types of cell stresses are accompanied by the activation of anti-stress genes that can suppress ROS synthesis.

The hypothalamus is activated in emergency situations. The hypothalamus stimulates the sympathetic nervous system, adrenal medulla and adrenal cortex. The adrenal medulla releases adrenaline and noradrenaline, while the adrenal cortex releases cortisol. Adrenaline (epinephrine) plays a central role in the stress reaction, and especially in the short-term stress reaction. The stress system relies on two key hormones: adrenaline and cortisol. Adrenaline works over a short time period, while cortisol has a prolonged period of action (Mark, 2007). In the present study, we investigated the influence of adrenaline and dexamethasone (a homolog of hydrocortisone) on ROS production by human neutrophils.

We hypothesized that different environmental factors affected organisms through changes in autologous serum (AS) protein structures. This affect was accomplished by the activation of anti-stress genes, followed by synthesis of Hsp70 molecules that increase cell resistance to oxidative stress. The goal of this work was to study the influence of heat and UV treatments on AS-induced ROS production by neutrophils isolated from the peripheral blood of donors in different age groups.

Our results demonstrate that the adsorption of AS onto neutrophils at $4^{\circ} \mathrm{C}$ (an unfavorable temperature for phagocytosis) prior to stimulation with opsonized zymosan enhanced ROS production; this was especially true for the adsorption of heated AS. These results coincide with data from other authors, who reported the stimulation of ROS production by human leukocytes independent of phagocytosis using heat-aggregated human IgG or serum-treated zymosan and cytochalasin B-treated neutrophils for the prevention of phagocytosis (Goldstein et al., 1975).

Some authors (Veloso et al., 2008) proposed that the inhibition of ROS production observed following treatment with autologous plasma was due to its antioxidant capacity. Our results did not show a marked antioxidant capacity of AS in patients of different ages following the treatment of neutrophils with $\mathrm{AS}$ at $4^{\circ} \mathrm{C}$ or their stimulation by AS at $37^{\circ} \mathrm{C}$.

According to our data the UV irradiation of AS caused changes in the stimulation of ROS production by neutrophils. A possible explanation is that the treatment could have results in changes in the structure of AS proteins. Indeed, UV irradiation has been shown to cause a reduction in human $\alpha$-lactalbumin by affecting the S-S bonds that form disulfide bridges (Permyakov et al., 2003).

The negative correlation between intracellular ROS production and Hsp70 found in this article may indicate that a higher concentration of Hsp70 in plasma is protective against oxidative stress. This higher concentration may cause lower levels of intracellular ROS production (Njemini et al., 2007).

In our investigations reduction in ROS production was observed after treatment of neutrophils with adrenaline at concentrations 10-4 and 10-5 M. This effect may be associated with both the activation function of the anti-stress genes by adrenaline and its influence as an antioxidant (Shimizy et al., 2010).

\section{Materials and Methods}

\section{Participants}

A total of 28 donors aged 38-59 years (three men), 17 donors aged 60-75 years (two men) and 14 long-lived donors aged 90 and over (two men) registered as patients in the Moscow Clinical Centre of Gerontology were recruited for the study. These donors suffered from co-morbidities, with coronary heart diseases cardiosclerosis, arterial hypertension, and cerebrovascular atherosclerosis diagnosed as the main pathologies. The inclusion criteria for participation were the absence of active pathologies (history of acute infection, tumors, apoplexy, or myocardial infarction) and treatment with corticosteroids or high doses of non-steroidal anti-inflammatory drugs for all subjects, and independent living for elderly and non-agenarians. The study was approved by Ethics Committee of Russian National Research Medical University named after N.I. Pirogov. All participants gave their informed consent prior to the study.

\section{Neutrophil Isolation}

Neutrophils were isolated from the peripheral blood within $2 \mathrm{~h}$ after blood sampling. The samples were centrifuged at $500 \times g$ for $30 \mathrm{~min}$ at room temperature (RT) in a density gradient using PolymorphPrep separation medium (Axis-Shield, Sweden). Fractions containing neutrophils were collected. The cells were washed twice $(400 \times g, 15 \mathrm{~min})$ in Dulbecco's phosphate buffer saline (DPBS), resuspended in RPMI-1640 media (SigmaAldrich, USA) supplemented with $2 \mathrm{mM} \mathrm{L}$-glutamine, $15 \mathrm{mM}$ HEPES and $2 \%$ fetal calf serum (HyClone, Thermo Scientific, USA; referred to hereafter as assay media) at a concentration of $2 \times 10^{6}$ cells $/ \mathrm{ml}$ and left for $30 \mathrm{~min}$ prior to use in assays. Neutrophil purity was assessed by flow cytometry analysis and was routinely found to be $\geq 95 \%$. Cell viability determined by trypan blue staining was at least $97 \%$.

\section{ROS Measurement by Luminol-Amplified Chemiluminometry}

Reactive oxygen species production was assessed using the luminol-amplified chemiluminometric method (Allen and Loose, 1976). Neutrophils $\left(2 \times 10^{5}\right.$ cells/sample $)$ were stimulated with zymosan A (Sigma-Aldrich, USA) opsonized with a freshly prepared serum pool from 10 donors at a final concentration of $20 \mathrm{mg} / \mathrm{ml}$ to induce ROS production. The reaction was performed at $37^{\circ} \mathrm{C}$ in plastic tubes in colorless Hank's Balanced Salt Solution (HBSS; $200 \mu \mathrm{l}$ ) and $1 \mu \mathrm{M}$ luminol (Serva, Germany) in a volume of $400 \mu \mathrm{l}$. The level of chemiluminescence in the cell samples was 
measured using a 3603 chemiluminometer (Dialog Joint Venture, Russia). The number of light pulses per minute (cpm) was registered. The kinetics of the level of chemiluminescence was recorded for $30 \mathrm{~min}$. Spontaneous ROS production was measured before zymosan treatment as the initial count per minute (cpm) level. The maximal cpm level was used for the calculation of zymosan-induced ROS production in a sample. The experiments were performed in duplicate.

\section{Measurement of Intracellular ROS Production}

Intracellular ROS generation in neutrophils was determined using $2^{\prime}-7^{\prime}$-dichlorodihydrofluorescein diacetate (DCFHDA, Invitrogen, USA; Bass et al., 1983). The probe was added to neutrophils resuspended in assay media $(500 \mu \mathrm{l})$ at a $5 \mu \mathrm{g} / \mathrm{ml}$ final concentration. After incubation for $20 \mathrm{~min}$ at $37^{\circ} \mathrm{C}$, the cells were washed twice with DPBS at $4^{\circ} \mathrm{C}$. Then, fluorescence at $530 \mathrm{~nm}$ was measured in the neutrophils by flow cytometry on a BD FACSCalibur flow cytometer (San Jose, CA, USA) with excitation at $488 \mathrm{~nm}$.

\section{Heat Treatment and Intracellular Hsp70 Immunolabeling}

Neutrophils in assay media were dispensed into polypropylene tubes $\left(10^{6}\right.$ cells in $\left.500 \mu \mathrm{l}\right)$ and heated in a constant-temperature water bath at $43^{\circ} \mathrm{C}$ for $10 \mathrm{~min}$ (heat shock) or at $42^{\circ} \mathrm{C}$ for $1 \mathrm{~min}$ (short-term stress) followed by recovery period of $1 \mathrm{~h}$ at $37^{\circ} \mathrm{C}$. Intracellular levels of $\mathrm{Hsp} 70$ were determined by indirect immunofluorescent staining, followed by flow cytometry analysis. For intracellular labeling, the neutrophils were fixed and permeabilized in DPBS containing 2\% paraformaldehyde (Riedel-de Haen, Germany), 0.05\% BSA and 0.05\% Triton X-100 (Sigma-Aldrich, USA) at $37^{\circ} \mathrm{C}$ for $15 \mathrm{~min}$. The permeabilized neutrophils were treated in a $100 \mu \mathrm{l}$ volume with the primary HSP70-specific monoclonal antibody BRM22 (Sigma-Aldrich, USA) or HSP70-specific B-hybridoma supernatants at 1:100 dilutions for $30 \mathrm{~min}$ at RT, and then stained with secondary sheep anti-mouse IgG Fab-fragments conjugated with PE (SigmaAldrich, USA) for $30 \mathrm{~min}$ at RT. Each stage of labeling was followed by two washes with DPBS containing $0.2 \%$ BSA and $0.1 \%$ Triton X-100. The cells were finally resuspended in DPBS and analyzed by flow cytometry. Intracellular HSP70 levels were determined by means of fluorescence intensity (MFI) corrected for the background fluorescence of the negative controls. The level of Hsp70 production by neutrophils was used as an indicator of anti-stress gene functions. Hsp70 is a very conservative family of cytoprotective proteins that are specifically induced in response to several environmental stresses at the cellular level, including heat shock, cellular energy depletion, oxidative stress or inflammation. Intracellular Hsp70 prevents abnormal folding of newly synthesized polypeptides or assists in the repair of damaged proteins (Ogava et al., 2008).

\section{Variations of AS Interactions with Neutrophils}

Autologous serum was prepared from the donor peripheral blood by centrifugation to remove cellular components and fibrinogens. There were two variations of AS interactions with neutrophils.
AS In the first variation, prior to the chemiluminescence reaction the neutrophils were treated with different dilutions of serum for $30 \mathrm{~min}$ at $4^{\circ} \mathrm{C}$, followed by washes. In the second variation, $200 \mu \mathrm{l}$ of AS (1:10 dilution) was used directly in the chemiluminescence reaction as a stimulator of ROS production by neutrophils at $37^{\circ} \mathrm{C}$. This temperature represents a favorable condition for phagocytosis. Prior to the interaction with neutrophils, the AS was heated in a water bath $\left(100^{\circ} \mathrm{C}\right)$ for $30 \mathrm{~s}$ or irradiated by UV rays of 200-280 nm using a quartz lamp with a power setting of $8 \mathrm{~W}$.

A total of $100 \mu \mathrm{l}$ of normal or heated AS was added to $100 \mu \mathrm{l}$ of neutrophils $\left(2 \times 10^{5}\right.$ cells $)$ and incubated for $30 \mathrm{~min}$ at $4^{\circ} \mathrm{C}$, followed by centrifugation at $400 \times g$ at $4^{\circ} \mathrm{C}$ for $10 \mathrm{~min}$. The cells were resuspended in $100 \mu \mathrm{l}$ of colorless Hanks. Control neutrophils suspended in colorless Hanks without AS were centrifuged at $4^{\circ} \mathrm{C}$ for $10 \mathrm{~min}$ and resuspended in $100 \mu \mathrm{l}$ of colorless Hanks.

The chemiluminescence reactions were performed in plastic tubes in colorless Hanks with $\mathrm{Ca}^{++}$and $\mathrm{Mg}^{++}$using luminol (Sigma) at a concentration of $2.5 \mu \mathrm{g} / \mathrm{ml}$. A total of $100 \mu \mathrm{l}$ of neutrophils were added to plastic tubes with $200 \mu \mathrm{l}$ of Hanks solution and $150 \mu \mathrm{l}$ of luminol in the revolving drum of the chemiluminometer for $1 \mathrm{~h}$ at $37^{\circ} \mathrm{C}$; then, the cells were stimulated by the addition of heated or UV irradiated AS or opsonized zymosan. The control tubes were treated with UV irradiated or normal Hanks. AS (1:10 dilution) was used directly in the chemiluminescence reaction as a stimulator of ROS production in a $200 \mu \mathrm{l}$ volume. Prior to the interaction with neutrophils, the AS was heated in a water bath $\left(100^{\circ} \mathrm{C}\right)$ for $30 \mathrm{~s}$ or irradiated by UV rays $(200-280 \mathrm{~nm})$ using a quartz lamp with a power setting of $8 \mathrm{~W}$ for 7 or $14 \mathrm{~min}$.

\section{Treatment of Neutrophils with Hormones}

The reaction was performed in plastic tubes in colorless Hanks solution with $\mathrm{Ca}^{++}$and $\mathrm{Mg}^{++}$. The control tubes contained $200 \mu \mathrm{l}$ of Hanks solution and $150 \mu \mathrm{l}$ of luminol (5-Amino2,3-dihydro-1,4-phthalazinedione, Serva, Germany). A total of $100 \mu \mathrm{l}$ of the neutrophil suspension $\left(2 \times 10^{5}\right.$ cells $)$ was added to the experimental and control tubes. Then, adrenaline or dexamethasone was added to the experimental tubes at a concentration of $10^{-4}$ or $10^{-5} \mathrm{M}$ for $30 \mathrm{~min}$ at $37^{\circ} \mathrm{C}$ prior to the chemiluminescence reaction.

\section{Flow Cytometry}

Flow cytometry analysis was performed on a FACSCalibur flow cytometer (BD Biosciences, USA) equipped with 488 and $640 \mathrm{~nm}$ lasers and an appropriate set of detectors and filters. Neutrophils were identified and gated using forward and side light scatter. A minimum of 10,000 gated events was collected for each sample. Data were analyzed using CellQuest ver. 3.4 (BD Biosciences) and FlowJo version 7.6.5.

\section{Statistical Analysis}

Statistical analysis was performed using the $\mathrm{R}$ 3.0.2 statistical system (The $\mathrm{R}$ Foundation for Statistical Computing). The significance of the differences between two groups was attained 
using a $t$-test. Correlation analysis was performed using the procedure from the stats package for R. Results were considered statistically significant at $p \leq 0.05$.

\section{Results}

Our findings show that AS affects ROS production in a dose dependent manner which is seen from Figure 1, presenting influence of different AS dilutions on ROS production. More diluted AS (i.e., 1:40) resulted in reduced enhancement of ROS production.

Centrifugation and resuspension procedures have been reported to reduce the neutrophil response to zymosan and subsequent ROS production (Figure 1, control 1 and 2). Adsorption of normal and heated AS onto neutrophils caused an increase in ROS production at AS dilutions of 1:10 and 1:20 in comparison with control 1. All patients were divided into three groups: long-lived (A) - mean age 93 years, senile and elderly patients (B) - mean age 71.4 years, and middle and young patients (C) - mean age 38 years (Figure 2). Heated AS evoked more ROS production compared to normal AS $(p<0.01)$ for groups B and C. Interestingly, the stimulation of ROS production by zymosan after treatment of the neutrophils from the long-lived group with normal AS did not differ significantly from the control; however, ROS production in the cell samples from the long-lived group after treatment of the neutrophils with heated AS was higher than the corresponding values in groups B and C. Data presented in Figure 2 demonstrate that in long-lived group of patients effect of AS on the ROS production is higher than in mean age and young patients groups.

In the second series of experiments, we showed that normal AS stimulated ROS production by neutrophils to a greater

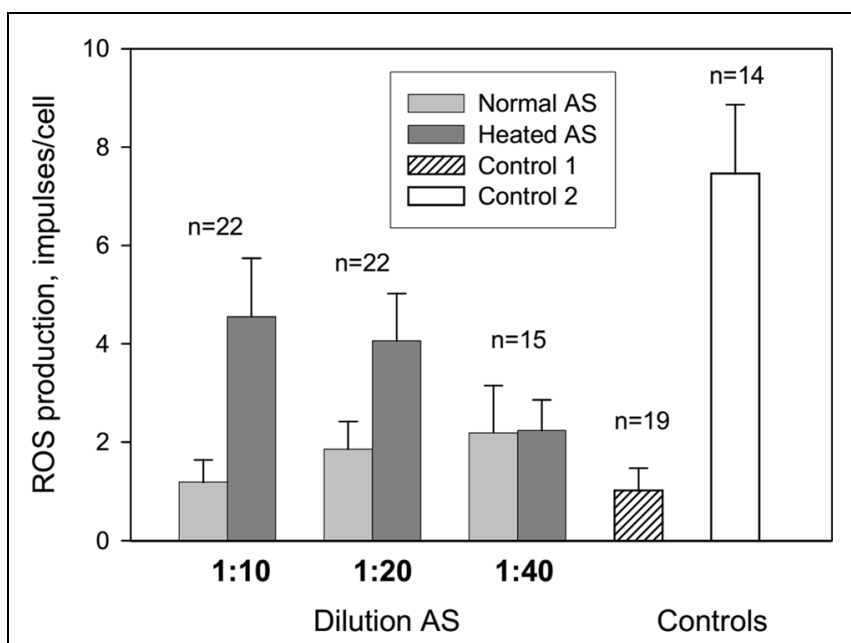

FIGURE 1 | Influence of autologous serum (AS) on reactive oxygen species (ROS) production by the patients' neutrophils measured by luminol-dependent chemiluminescence. Control 1: neutrophils in colorless Hanks without AS were centrifugated at $4^{\circ} \mathrm{C} 10$ min and resuspended in $100 \mathrm{ml}$ of colorless Hanks. Control 2: neutrophils in colorless Hanks have not centrifuged.
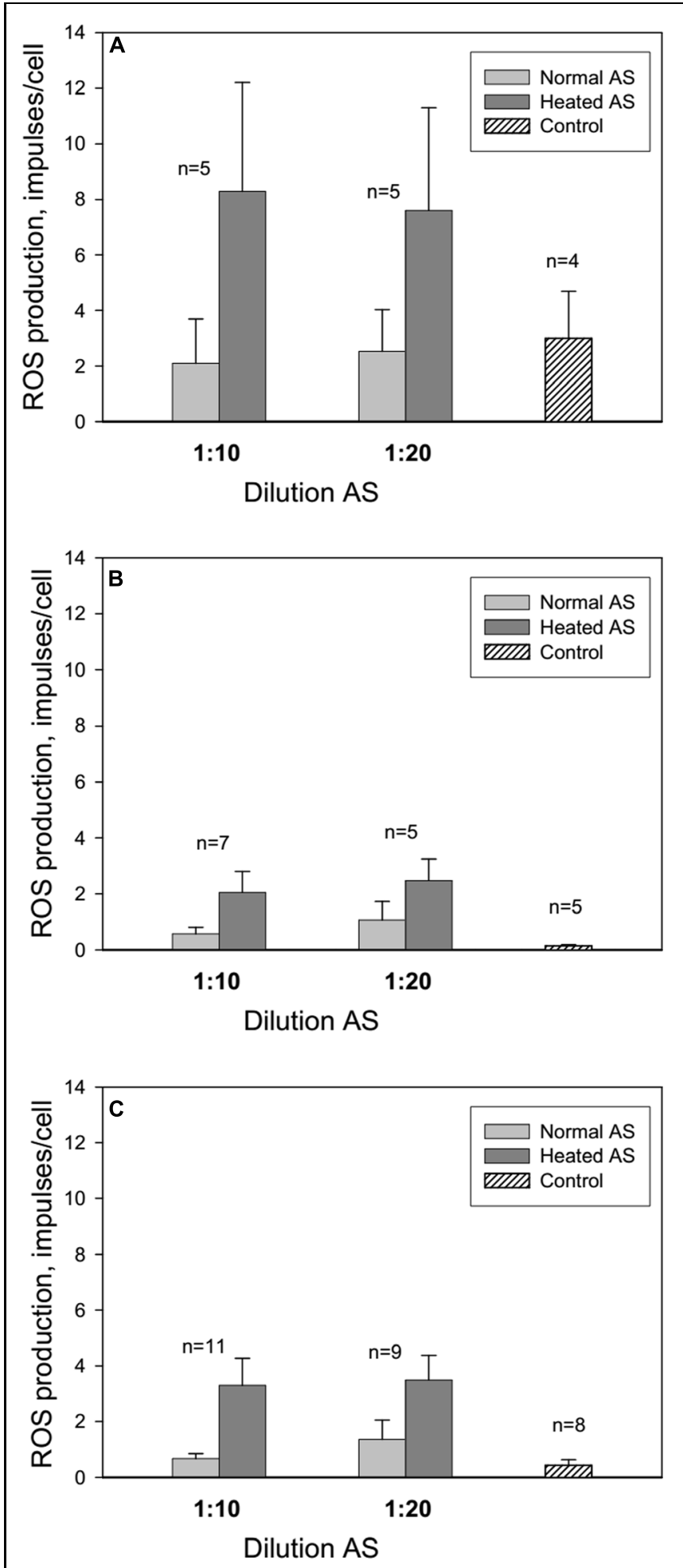

FIGURE 2 | Influence of AS on ROS production by neutrophils from long-lived patients above 90 years of age (A), patients between 60 and 75 years of age $(B)$ and middle aged patients from 40 to 59 years of age (C). Control: neutrophils in colorless Hanks without AS were centrifugated at $4^{\circ} \mathrm{C} 10 \mathrm{~min}$ and resuspended in $100 \mu \mathrm{l}$ of colorless Hanks. The line above the bar indicates the SD; $n-$ number of patients. 
extent than heated AS (Figure 3). UV irradiation of AS for 7 min induced ROS production by neutrophils to a greater extent than AS that was UV irradiated for $14 \mathrm{~min}$. UV irradiation of Hanks solution for 14 min did not influence ROS production.

Figure 4 shows the dynamics of ROS production from neutrophils collected from an 86 years old patient after stimulation of the cells by normal, heated or UV irradiated AS. The changes in ROS production were similar to the previous data presented in Figure 3. Involvement of the heat shock function or anti-stress genes of neutrophils in response to treatment with the different types of AS and zymosan may be determined based on the stress impact on cells prior to their stimulation in the chemiluminescence reaction. Heating stress reduces ROS

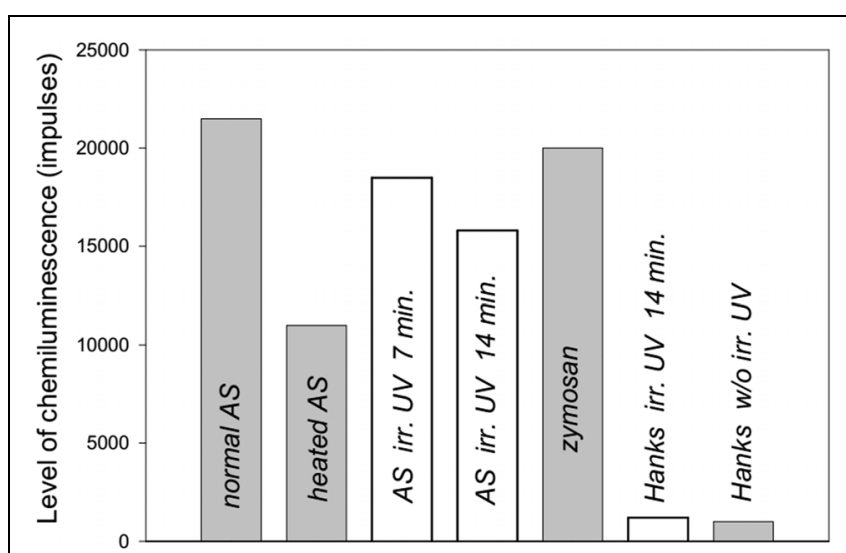

FIGURE 3 | Level of ROS production from human neutrophils stimulated by heated or ultraviolet (UV) irradiated AS (collected from a 38-years-old patient) measured by luminol-dependent

chemiluminescence. The maximal levels of induced chemiluminescence of neutrophils are presented.

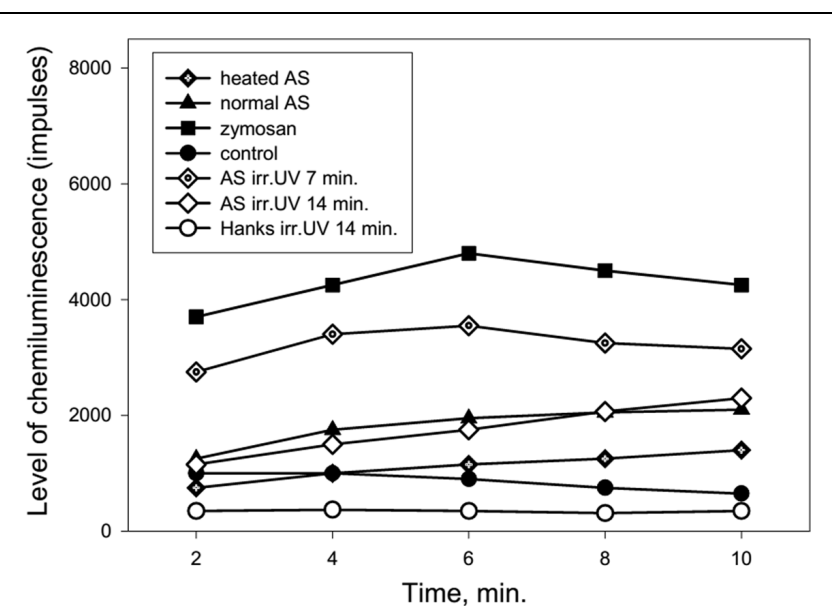

FIGURE 4 | Dynamics of ROS production by human neutrophils stimulated by heated or UV irradiated AS (collected from an

86-years-old patient). AS was heated in a water bath $\left(100^{\circ} \mathrm{C}\right)$ for $30 \mathrm{~s}$ or irradiated by UV (200-280 nm) using the quartz lamp with a power setting of $8 \mathrm{~W}$ for 7 or 14 min prior to neutrophil stimulation. production as can be seen from Figure $\mathbf{5}$ from dynamics of ROS production following the stimulation of heated AS by neutrophils after cells stress. UV irradiation of AS induces ROS production in time which is shown in Figure 5.

Figure 5 shows that heat shock (accomplished by heating the neutrophils for $1 \mathrm{~min}$ at $42^{\circ} \mathrm{C}$ ) caused a marked reduction of ROS production by neutrophils in response to treatment with normal, heated or UV irradiated AS.

When neutrophils from the long-lived and 60- to 89-years-old patients were exposed to heat shock, the quantity of intracellular Hsp70 was negatively correlated with the level of intracellular ROS (Figure 6). Moreover, zymosan-induced extracellular ROS production showed a negative correlation with the level of intracellular ROS in the long-lived group (Figure 7A) that was absent in the 60- to 89-years-old patients (Figure 7B).

The treatment of neutrophils collected from patients in the different age groups with adrenaline at concentrations of $10^{-4}$ and $10^{-5} \mathrm{M}$ (Figures 8-10) caused a reduction in ROS production. In contrast, treatment with dexamethasone (from the other stress hormone group) at the same concentration did not evoke this effect (Figures 9 and 10).

\section{Discussion}

An autologous system is more closely related to physiological processes at the level of a whole organism. It is important to study the concrete mechanisms behind the regulation of ROS production by human phagocytes. Our data demonstrate that the adsorption of AS onto neutrophils at $4^{\circ} \mathrm{C}$ prior to stimulation with opsonized zymosan enhanced ROS production; this was especially true for the adsorption of heated AS. The adsorption of AS onto neutrophils at $4^{\circ} \mathrm{C}$ represents an unfavorable condition

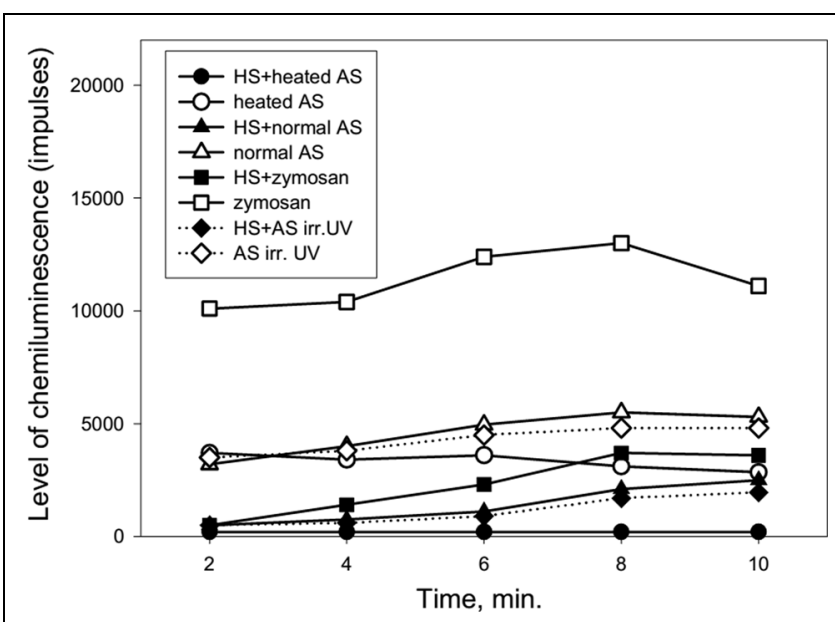

FIGURE 5 | Dynamics of ROS production following the stimulation of heated or UV irradiated AS by neutrophils after cells stress (collected from a 92-years-old patient). AS was heated in a water bath $\left(100^{\circ} \mathrm{C}\right)$ for $30 \mathrm{~s}$ or irradiated by UV (200-280 nm) for $14 \mathrm{~min}$. The stress impact was evoked by heating the neutrophils at $42^{\circ} \mathrm{C}$ for $1 \mathrm{~min}$ followed by a recovery period of $1 \mathrm{~h}$ at $37^{\circ} \mathrm{C}$ prior to cell stimulation. 

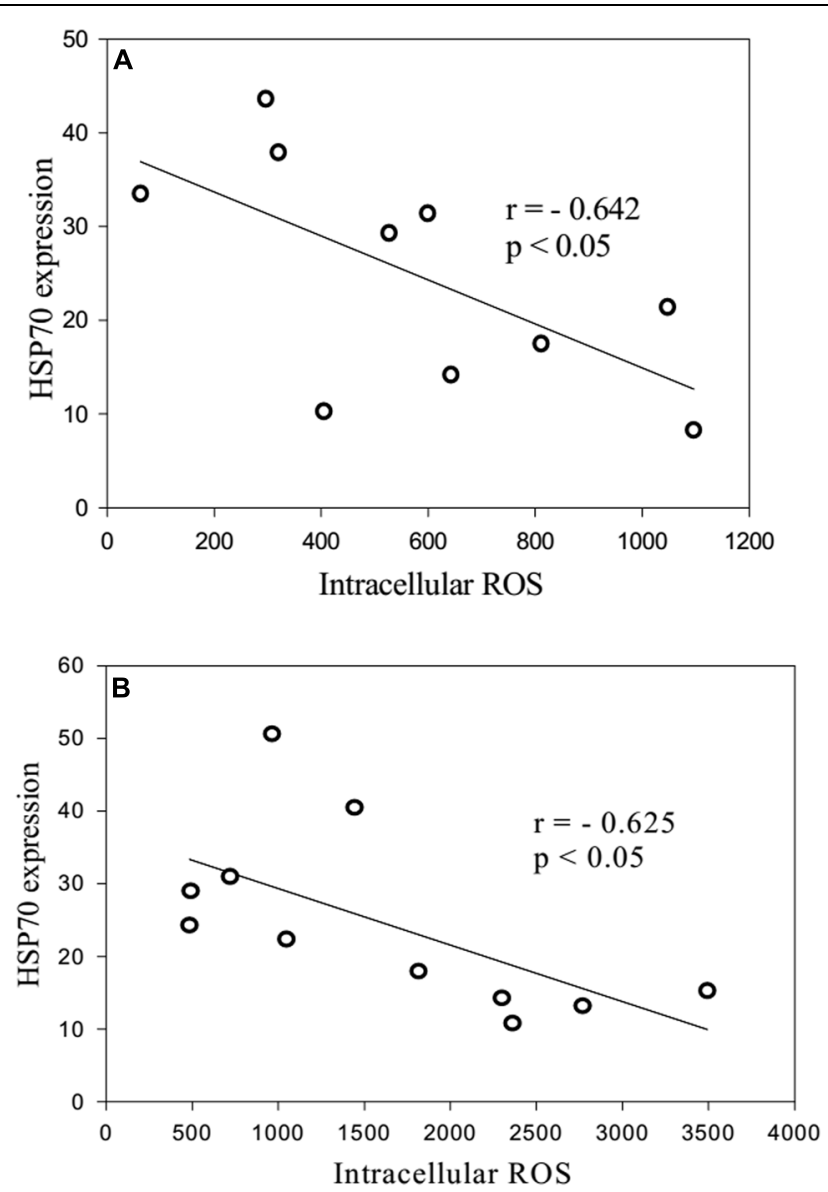

FIGURE 6 | Correlation between the quantity of intracellular HSP70 after stress and the levels of intracellular ROS production from heat shock-exposed neutrophils [(A): long-lived patients; (B) 60- to 89-years-old patients].

for phagocytosis. The results of our examination coincide with data from other authors, who reported the stimulation of ROS production by human leukocytes independent of phagocytosis using heat-aggregated human IgG or serum-treated zymosan and cytochalasin B-treated neutrophils for the prevention of phagocytosis (Goldstein et al., 1975). The interaction of aggregated serum proteins with neutrophil Fc receptors may be caused by the increasing ROS production by the cell mitochondria after stimulation with opsonized zymosan through the $\mathrm{C} 3 \mathrm{~b}$ receptor. Our results attach importance to the quality of serum proteins, because the use of more dilute AS (i.e., 1:40) resulted in a reduced enhancement of ROS production. High concentrations of proteins in AS can reduce the function of antistress or heat shock genes and the synthesis of the HSPs that suppress ROS production. HSP70 can inhibit the main producer of ROS synthesis (NADPH oxidase) in human neutrophils, and thereby suppress ROS production (Maridonneau-Parini et al., 1988). The results that in long lived group of patients effect of AS on the ROS production is higher than in mean age and young patients groups can point that with age the conformal
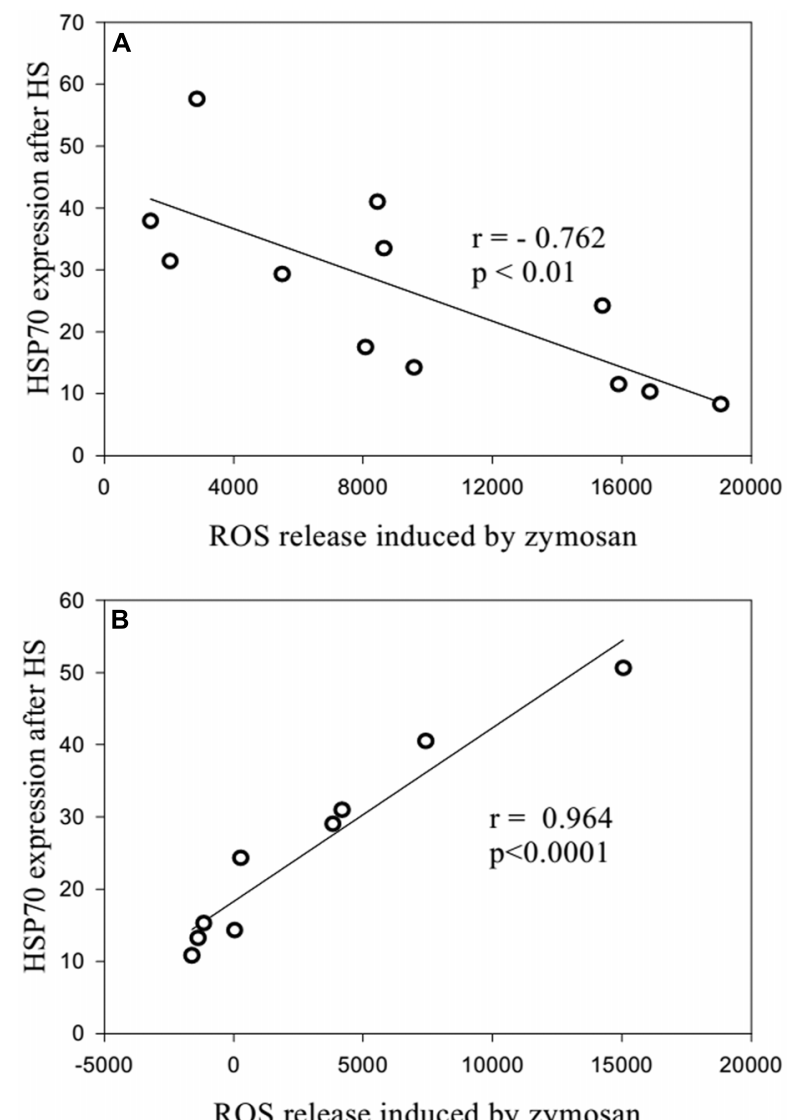

FIGURE 7 | Correlation between the quantity of intracellular HSP70 after stress and the levels of zymosan-stimulated intracellular ROS production [(A): long-lived patients; (B) 60- to 89-years-old patients].

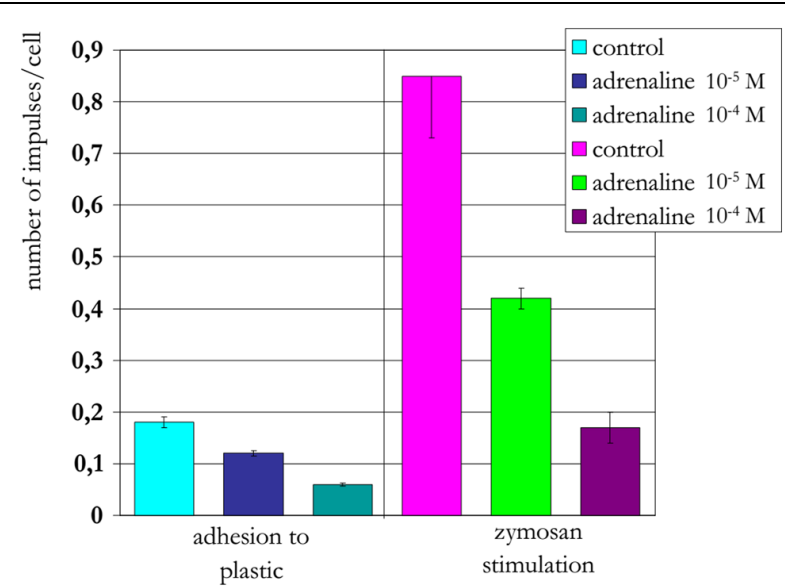

FIGURE 8 | Influence of adrenaline on ROS production by neutrophils from a 22-years-old patient (induced by chemiluminescence).

changes of proteins increase and anti-stress genes functions decrease. These can be considered as a biological indicator of senescence. 


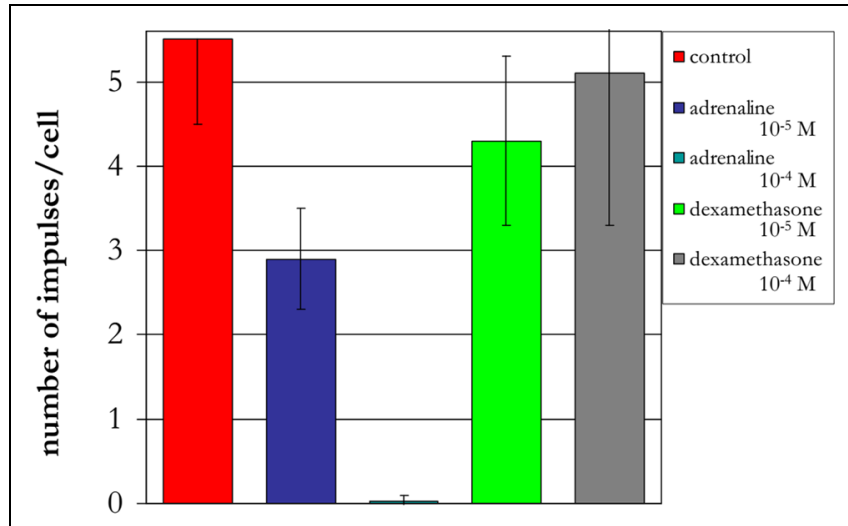

stimulation by zymosan

FIGURE 9 | Influence of adrenaline and dexamethasone on ROS production by neutrophils from a 90 -years-old patient (induced by chemiluminescence).

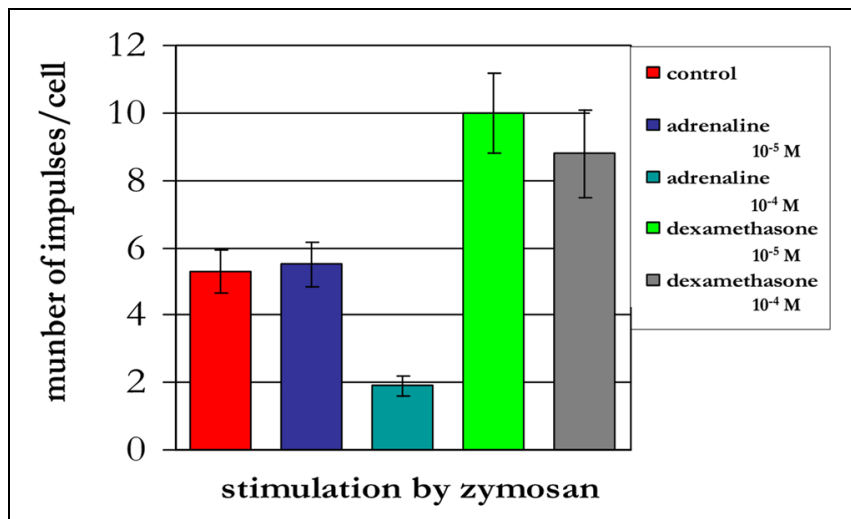

FIGURE 10 | Influence of adrenaline and dexamethasone on ROS production by neutrophils from a 94-years-old patient (induced by chemiluminescence).

In our previous work (Ponomarev et al., 2005), we demonstrated reduced ROS production by human neutrophils by the addition of recombinant HSP70 in a luminol-dependent chemiluminescence assay after stimulation with opsonized zymosan. The stress impact on neutrophils from patients in the different age groups following heating for $1 \mathrm{~min}$ at $42^{\circ} \mathrm{C}$ resulted in the reduction of ROS production in response to opsonized zymosan and normal, heated or UV irradiated AS.

The use of normal or heated AS for the stimulation of ROS production by neutrophils at $37^{\circ} \mathrm{C}$ (representing the favorable condition for phagocytosis) differed from the results of the treatment of neutrophils with the same types AS at $4^{\circ} \mathrm{C}$. The heated AS caused a reduction in ROS production compared with normal AS. This finding may be attributed to the phagocytosis of the aggregated heated AS proteins following the activation of heat shock genes and synthesis of HSPs and the suppression of ROS production. Other authors have demonstrated the inhibition of ROS production in peripheral blood mononuclear cells from healthy and type 2 diabetic patients following treatment with autologous plasma through the Akt/PKB signaling phosphorylation pathway using luminol-dependent chemiluminescence (Veloso et al., 2008). The authors proposed that the inhibition of ROS production observed following treatment with autologous plasma was due to its antioxidant capacity. Our results did not show a marked antioxidant capacity of AS in patients of different ages following the treatment of neutrophils with AS at $4^{\circ} \mathrm{C}$ or their stimulation by $\mathrm{AS}$ at $37^{\circ} \mathrm{C}$. ROS production following the treatment of neutrophils with normal AS did not differ from the control in centenarians, probably due to the preservation of some of the antioxidant activity of normal AS. However, ROS production following treatment of neutrophils with normal AS differed significantly from the controls in the senile and elderly and the middle and young patients groups.

The finding that normal AS stimulated ROS production by neutrophils to a greater extent than heated AS may be attributed to the phagocytosis of the aggregated heated AS proteins following the activation of heat shock genes and synthesis of HSPs and the suppression of ROS production. The UV irradiation of AS may have caused changes in the stimulation of ROS production by neutrophils due to its effect on the complement C3 factor and the subsequent enhancement of phagocytosis (Artjuhov et al., 2005). An alternative explanation is that the treatment could have results in changes in the structure of AS proteins. Indeed, UV irradiation has been shown to cause a reduction in human $\alpha$-lactalbumin by affecting the $S-S$ bonds that form disulfide bridges (Permyakov et al., 2003).

Our data demonstrate the possible influence of the phagocytosis of AS proteins with altered structures by neutrophils on HSG and HSP production, which can suppress synthesis of ROS. Human aging may be associated with a decrease in the functional activity of HSGs and phagocytosis. As a consequence of the decline in the neutrophils HSP response, the suppression of ROS generation is weakened and phagocytes produce high level of ROS within regions of inflammation (Ogava et al., 2008), thereby injuring self tissues. The functional activity of HSGs and the synthesis of HSP70 can affect the level of regulation of gene transcription by heat shock factors (Singh et al., 2007). Cellular senescence may be evoked through enhanced synthesis of ROS by phagocytes as a result of frequent stresses in addition to the prominent reduction in the function of anti-stress genes and the synthesis of HSPs. Damaging environmental factors can induce cell stress in an organism by acting on AS proteins of ("serum" mechanism of aging; Semenkov et al., 2005).

The reduction of the excessive concentration of ROS that results from oxidative stress can be achieved by antioxidant treatment, such as ascorbic acid (vitamin C), glutathione, melatonin, superoxide dismutase, and catalase. The treatment should be focused on the individual sensitivity of the patients, which is reflected in the decrease of ROS levels to normal values and the need of the individual to control the current ROS level (Maridonneau-Parini et al., 1988; Ponomarev et al., 2005; Veloso et al., 2008). 
Ultraviolet radiation is an important factor involved in premature aging. The role of sunlight in the process of premature aging is so significant that it has been called "photoaging." In addition to serving as a source for ionizing radiation, UV light may cause the development of oxidative stress in humans. AS protein structures changed by heating or irradiation by UV rays $(200-280 \mathrm{~nm} 8 \mathrm{~W})$ for $10 \mathrm{~min}$ resulted in decreased neutrophil ROS production by means of phagocytosis and the activation of anti-stress genes, which control Hsp70 production. We propose that the effect of environment factors on AS proteins can cause an adverse increase in oxidative stress levels due to the functional reduction in anti-stress gene expression.

The negative correlation between intracellular ROS production and Hsp70 may indicate that a higher concentration of Hsp70 in plasma is protective against oxidative stress, which may cause lower levels of intracellular ROS production (Njemini et al., 2007). Our previous results showed the dependence of the correlation on the synthesis of Hsp70 and ROS production on the patient's age (Kovalenko et al., 2014).

There was a negative correlation between zymosan-induced extracellular ROS production and the level of intracellular ROS in the long-lived group that was absent in the 60- to 89-yearsold patients. The determination of different types of ROS (i.e., intracellular hydrogen peroxides and extracellular superoxide anions) should be taken into account. These data showed some possible functional defects in the ability of intracellular Hsp70 to suppress extracellular ROS production in elderly and senile patients with polymorbidity.

Short-term heating stress ( $1 \mathrm{~min}$ or less) at $42^{\circ} \mathrm{C}$ was followed by a prominent reduction in ROS production (Semenkov et al., 2014). Under similar conditions, the same effect was observed after treatment of neutrophils with adrenaline at concentrations $10^{-4}$ and $10^{-5} \mathrm{M}$. The reduction in ROS production may be associated with both the activation function of the antistress genes by adrenaline and its influence as an antioxidant (Shimizy et al., 2010). In contrast, dexamethasone from the other stress hormone group did not evoke the same type of effect

\section{References}

Allen, R. C., and Loose, L. D. (1976). Phagocytic activation of a luminoldependent chemiluminescence in rabbit alveolar and peritoneal macrophages. Biochem. Biophys. Res. Commun. 69, 245-252. doi: 10.1016/S0006-291X(76) 80299-9

Artjuhov, V. G., Gusinskaja, V. V., and Michaleva, E. A. (2005). Interaction of neutrophils of human's blood with $\mathrm{C} 3$ factor complement in condition of ultraviolet irradiation. Immunology 2, 76-79.

Bass, D. A., Patce, J. W., Dechatelet, L. R., Szejda, P., Seeds, M. C., and Thomas, M. (1983). Flow cytometric studies of oxidative product formation by neutrophils: a graded response to membrane stimulation. J. Immunol. 130, 1910-1917.

Goldstein, I. M., Roos, D., Kaplan, H. B., and Weissmann, G. (1975). Complement and immunoglobulins stimulate superoxide production by human leukocytes independently of phagocytosis. J. Clin. Invest. 56, 1155-1163. doi: 10.1172/JCI108191

Kovalenko, E. I., Boyko, A. A., Semenkov, V. F., Lutsenko, G. V., Grechikhina, M. V., Kanevskiy, L. M., et al. (2014). ROS production, intracellular HSP70 when provided at the same concentration. Dexamethasone is a homolog of hydrocortisone, which can suppress HSG function and HSP production.

\section{Conclusion}

In this article, we studied the influence of AS on ROS production in neutrophils isolated from the peripheral blood of donors in different age groups. The presented results show a new mechanism by which some environment factors (e.g., high temperature and UV light) impact oxidative stress inductors by changing the structure of AS proteins. It is known from the literature that after heating at $100^{\circ} \mathrm{C}$ proteins lose the quaternary structure while UV irradiation may damage disulfide bridge in proteins. These proteins affect the functions of the anti-stress genes that control anti-stress protein synthesis (Hsp70). The interaction of AS treated with heat or UV light with neutrophils evoked a reduction in ROS production at a temperature that was favorable for phagocytosis $\left(37^{\circ} \mathrm{C}\right)$.

A negative correlation was found between the quantity of intracellular Hsp70 and the level of intracellular ROS production. This negative correlation was observed following $10 \mathrm{~min}$ of heat shock at $43^{\circ} \mathrm{C}$. Short-term heating stress $(1 \mathrm{~min})$ at $42^{\circ} \mathrm{C}$ was followed by a prominent reduction in ROS production. The same effect was observed after treatment of neutrophils with adrenaline at concentrations of $10^{-4}$ and $10^{-5} \mathrm{M}$. Dexamethasone from the other stress hormone group did not evoke the same effect when provided at the same concentration.

The authors hope that the results of the investigation will be useful for future research into the influence of environment damage on anti-stress gene functions by means of AS proteins.

\section{Funding}

The research was supported by The International Scientific and Technology Center (grant 3303).

levels and their relationship in human neutrophils: effects of age. Oncotarget 5, 11800-11812.

Maridonneau-Parini, I., Clerc, J., and Polla, B. S. (1988). Heat shock inhibits NADPH oxidase in human neutrophils. Biochem. Biophys. Res. Commun. 154, 179-186. doi: 10.1016/0006-291X(88)90667-5

Mark, P. E. (2007). Mechanisms and clinical consequences of clinical illness associated adrenal insufficiency. Curr. Opin. Crit. Care 13, 363-369. doi: 10.1097/MCC.0b013e32818a6d74

Njemini, R., Bautmans, I., Lambert, M., Demanet, C., and Mets, T. (2007). Heat shock proteins and chemokine/citokine secretion profile in aging and inflamation. Mech. Ageing Dev. 128, 450-454. doi: 10.1016/j.mad.2007. 06.003

Ogava, K., Suzuki, K., Okutsu, M., Yamazaki, K., and Shinkai, S. (2008). The association of elevated reactive oxygen levels from neutrophils with low-grade inflammation in the elderly. Immun. Ageing 5, 13-20. doi: 10.1186/1742-49335-13

Penke, L., Drost, E., Mac Nee, W., Deury, L. J., and Starr, J. M. (2011). Skin ageing and oxidative stress in a narrow-age cohort of older adults. Eur. Geriatr. Med. 2, 140-146. doi: 10.1016/j.eurger.2011.04.009 
Permyakov, E. A., Permyakov, S. E., Deikus, G. Y., Morozova-Roche, L. A., Grischenko, V. M., et al. (2003). Ultraviolet illumination-induced reduction of $\alpha$-lactalbumin disulfide bridges. Proteins 51, 498-503. doi: 10.1002/prot.10371

Ponomarev, A. D., Semenkov, V. F., and Sapozhnikov, A. M. (2005). Influence of heat shock proteins on production of reactive oxygen species by human neutrophils. Immunologia 2, 72-75.

Semenkov, V. F., Karandashov, V. I., and Kovalchuk, L. V. (2005). Immunogerontology: A Manual for Physician. Moscow: Meditsina Publishers.

Semenkov, V. F., Michalski, A. I., Grechikhina, M. V., and Beliakov, B. K. (2014), Method of Measurement of Individual Human Sensitivity to Oxidative Stress. R.F. Patent No 2508545. Moscow: Rospatent.

Shimizy, T., Nakanishi, Y., Nakahare, N., Wada, N., Moro-oka, Y., Hiranj, N., et al. (2010). Structure effect on abtioxidant activity of catechlamines towards singlet oxygen and other reactive oxygen species in vitro. J. Clin. Biochem. Nutr. 47, 181-190. doi: 10.3164/jcbn.09-112

Singh, R., Kolvraa, S., and Rattan, S. I. S. (2007). Genetics of human longevity with emphasis on the relevance of HSP70 as candidate genes. Front. Biosci. 12:4504-4513. doi: 10.2741/2405
Veloso, C. A., Isoni, C. A., Borges, E. A., Mattos, R. T., Calsolari, M. R., Reis, J. S., et al. (2008). Inhibition of ROS production in peripheral blood mononuclear cells from type 2 diabetic patients by autologous plasma depends on Akt/PKB signaling pathway. Clin. Chim. Acta 394, 77-80. doi: 10.1016/j.cca.2008. 04.010

Conflict of Interest Statement: The Associate Editor, Elena Pasyukova, declares that, despite being affiliated to the same institution as the authors Anatoli Michalski and Alexander Sapozhnikov, the review process was handled objectively. The authors declare that the research was conducted in the absence of any commercial or financial relationships that could be construed as a potential conflict of interest.

Copyright $\odot 2015$ Semenkov, Michalski and Sapozhnikov. This is an open-access article distributed under the terms of the Creative Commons Attribution License (CC BY). The use, distribution or reproduction in other forums is permitted, provided the original author(s) or licensor are credited and that the original publication in this journal is cited, in accordance with accepted academic practice. No use, distribution or reproduction is permitted which does not comply with these terms. 